\title{
Iron Overload in Patients with Chronic Lymphocytic Leukemia Complicated by Autoimmune Hemolytic Anemia: A Case Report
}

\author{
Mohammad Alia ${ }^{a}$ Mohamed A. Yassin ${ }^{b}$ \\ aDepartment of Medical Education, Hamad Medical Corporation, Doha, Qatar; ${ }^{b}$ Department \\ of Medical Oncology, Hematology Section, National Center for Cancer Care \& Research, \\ Hamad Medical Corporation, Doha, Qatar
}

\section{Keywords}

Chronic lymphocytic leukemia · Autoimmune hemolytic anemia - Liver iron concentration

\begin{abstract}
Iron overload is commonly seen in the context of hematological diseases in patients who require frequent transfusions. On the other hand, an association between autoimmune hemolytic anemia complicating chronic lymphocytic leukemia and iron overload is unusual. Here, we report 2 cases of iron overload in patients with a background of chronic lymphocytic leukemia complicated by autoimmune hemolytic anemia. Both patients were found to have a high liver iron concentration on liver MRI (FerriScan). This observation raises the important question about the value of screening for iron overload in this group of patients.

\section{Introduction}

Chronic lymphocytic leukemia (CLL) is one of the most common types of leukemia in Western countries. It is characterized by the progressive accumulation of usually monoclonal, functionally incompetent lymphocytes. It can affect B and T lymphocytes; however, the majority of diagnosed cases are due to accumulation of monoclonal B cells. These cells will primarily accumulate in the bone marrow and blood [1].

Patients with CLL commonly develop complications associated with intrinsic immune dysfunction caused by the accumulation of these incompetent lymphocytes, resulting in immunodeficiency and the development of autoimmune disorders.
\end{abstract}


One of the most common autoimmune complications in CLL patients is autoimmune hemolytic anemia (AIHA). There are no accurate data about the incidence of AIHA among CLL patients, although most series suggest a rate of 5-10\% [2].

Patients with AIHA will usually have an isolated drop in hemoglobin levels and a positive direct antiglobulin (Coombs) test result. Other common laboratory findings include an increase in reticulocyte count, indirect hyperbilirubinemia, reduced haptoglobin levels, and an increase in serum lactate dehydrogenase levels.

Iron overload is commonly seen in patients with hematological malignancies requiring repeated transfusions [3]. However, it has not been reported before in patients with CLL and AIHA not requiring blood transfusion, which raises the question about the importance of screening this group of patients.

Here, we report on 2 patients with CLL and AIHA who had never received any blood transfusion and were found to have iron overload.

\section{Case Presentation}

The first patient was a 29-year-old gentleman presenting with anemia manifesting as a drop in hemoglobin levels from 14 to $6 \mathrm{~g} / \mathrm{dL}$. A workup revealed AIHA, and the patient was treated with a course of steroids but did not receive any blood transfusion. After a few months, he was diagnosed with CLL and required treatment for count doubling; thus, he received 6 cycles of fludarabine, cyclophosphamide, and rituximab and achieved complete remission. His ferritin level after chemotherapy was $1,300 \mu \mathrm{g} / \mathrm{L}$, and MRI showed a liver iron concentration (LIC) of $9.8 \mathrm{mg} / \mathrm{g}$ dry tissue (Fig. 1).

Fig. 1. FerriScan of the first patient before chelation, with a liver iron concentration of $9.8 \mathrm{mg} / \mathrm{g}$ dry tissue.

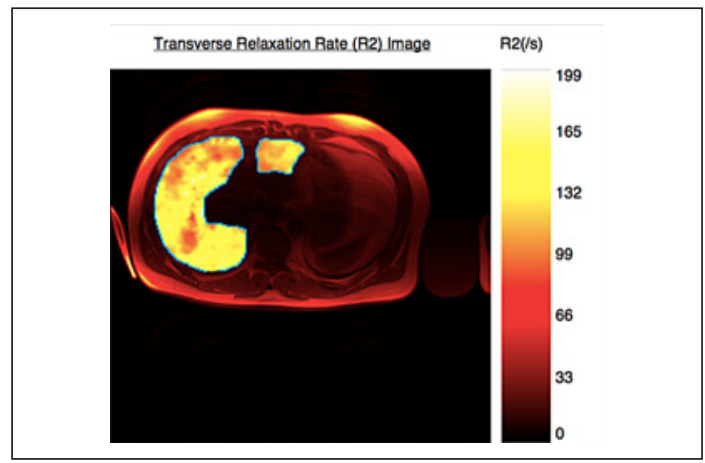

The patient was started on the iron chelating agent deferasirox (DFX; Exjade) but could not tolerate it because of gastrointestinal side effects; thus, he was shifted to the new formula of DFX (Jadenu). He received a dose of $1,080 \mathrm{mg}$ for 1 year, which he tolerated with no toxicity. MRI was repeated 1 year later, and it showed a normal LIC (Fig. 2).

Fig. 2. FerriScan of the first patient after chelation, with a liver iron concentration of $2.3 \mathrm{mg} / \mathrm{g}$ dry tissue.

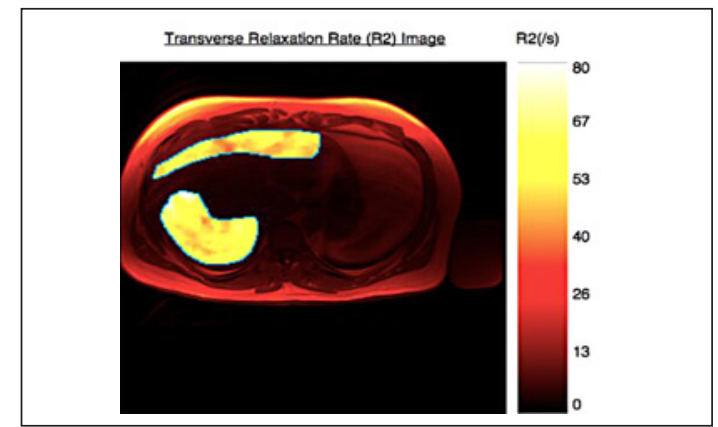


The second patient was a 70-year-old gentleman with CLL stage 0 monitored by watchful waiting. He presented with a drop in hemoglobin levels from 12 to $4 \mathrm{~g} / \mathrm{dL}$ and was diagnosed with AIHA. He was treated with steroids but did not receive any blood transfusion. After recovery, MRI showed an LIC of $6.2 \mathrm{mg} / \mathrm{g}$ dry weight. The patient received DFX (Exjade) at a dose of $30 \mathrm{mg} / \mathrm{kg}$, and 6 months later, his LIC was within normal limits.

\section{Discussion}

Iron is stored in the body in the form of a ferritin complex, which is most commonly found in the liver, bone marrow, and spleen. However, the liver is the main storage reservoir, estimated to contain $70 \%$ of the total iron of the body [4].

In the context of hematological diseases, iron is deposited in the body via different mechanisms. The most common mechanism is iron deposition in the body due to lifelong blood transfusion, as seen in individuals with beta-thalassemia major [4]. Another mechanism is seen in patients with non-transfusion-dependent sickle cell disease, where iron is deposited in the body due to chronic hemolysis [5]. On the other hand, individuals with beta-thalassemia intermedia develop iron overload due to defective hemoglobin synthesis, leading to an increase in the absorption of iron from the gastrointestinal tract [6].

Iron overload has also been reported in patients with myelodysplastic syndromes (MDS) and acute leukemia requiring repeated blood transfusions [3]. Besides repeated blood transfusions, iron overload in patients with MDS is thought to result from suppression of the hepcidin level, causing an increase in iron absorption from the gastrointestinal tract. Moreover, individuals with MDS can develop iron overload due to an underlying genetic defect. SF3B1 gene mutation in patients with MDS causes a further reduction in the hepcidin-to-ferritin ratio [7]. SF3B1 is a gene encoding a component of the RNA splicing machinery, and in one study, a mutation of this gene was found in $28 \%$ of the MDS patients [8].

Iron overload is more prevalent among patients with acute than among those with chronic leukemia, due to the former patients' greater requirement for transfusion [3]. However, iron overload has not been reported before in patients with CLL and AIHA not requiring blood transfusion. Moreover, during our literature review for an association between AIHA and iron overload, we found only a single case of iron overload in a patient with Evans syndrome presenting with a severe AIHA crisis requiring blood transfusion of 20 units [9]. This case is different from the 2 cases we have been reporting on, since both of our patients did not receive any blood transfusion.

Patients with iron overload usually do not exhibit any symptoms initially until the excess iron deposition in the body's organs leads to complications. Excess iron may be deposited in the liver, heart, and endocrine organs. Excess iron deposition can lead to liver cirrhosis and heart failure. In addition, various endocrine complications can result from iron overload; one study found a higher prevalence of glucose dysregulation among patients with acute and chronic hematological malignancies requiring repeated transfusions, and fasting blood glucose was correlated significantly with the amount of iron transfused as well as with ALT levels [3]. Moreover, thyroid dysfunction is a known complication of iron overload; a study done on patients with beta-thalassemia major showed worsening of thyroid function in $35 \%$ of the study population by the age of 18 years [10]. Another study showed that $7.6 \%$ of their patients with thalassemia major developed central hypothyroidism [11].

Other endocrine complications include short stature and hypogonadotropic hypogonadism, as observed in a study on 28 adolescent patients with thalassemia intermedia; 
$25 \%$ of the patients in the study had short stature, and $10.7 \%$ had hypogonadotropic hypogonadism, with no difference between nontransfused and infrequently transfused patients [6]. The association between iron overload and short stature is supported by another study showing that $50 \%$ of their thalassemia major patients had IGF-1 2 SD below the average value for healthy individuals [12]. Besides that, iron overload and chronic liver disease play important roles in the development of a hypogonadotropic organism according to a study done on 11 patients with thalassemia major and hypogonadotropic hypogonadism [13].

The gold standard method for assessing iron overload, in the absence of liver cirrhosis, is the LIC as determined by liver biopsy. A standardized MRI method (FerriScan) is a reasonable noninvasive method for assessing the LIC with high sensitivity and specificity ( 94 and $100 \%$, respectively) [7]. The normal LIC is between 0.4 and $2.2 \mathrm{mg} / \mathrm{g}$ dry liver weight; an LIC level up to $7 \mathrm{mg} / \mathrm{g}$ dry liver weight is not associated with significant adverse effects, while values $>15 \mathrm{mg} / \mathrm{g}$ are being consistently associated with liver cirrhosis [5].

Iron chelation is proven to decrease organ dysfunction and improve survival among patients with transfusion-dependent anemias. There are three major classes of iron chelators: deferoxamine (DFO), deferiprone, and DFX. Several studies have shown heightened compliance with the oral chelators DFX and deferiprone compared to DFO, due to their ease of use compared to DFO (subcutaneous or IV injection). Moreover, one study established that the efficacy of oral DFX is comparable to that of standard iron chelators [14].

Exjade and the new tablet Jadenu are two different DFX formulations. Exjade must be taken on an empty stomach and it should be mixed with water, apple juice, or orange juice. It is unpalatable and associated with gastrointestinal discomfort. According to one study, Jadenu was associated with improvement in palatability compared to Exjade; only mild gastrointestinal symptoms in 3 out of the 12 patients enrolled in the study, who did not require discontinuation of therapy; and no other side effects [15]. Here, we made a similar observation, since the first patient had better tolerability when treatment was shifted to Jadenu, without any gastrointestinal side effects.

A preliminary report on Jadenu's effect on the LIC and its biochemical profile published in 2018 showed a nonsignificant decrease in LIC and serum ferritin levels after 1 year of treatment [15]. We made a different observation, as the LIC of the first patient decreased significantly from 9.8 to $2.3 \mathrm{mg} / \mathrm{g}$ dry tissue after 1 year of treatment with Jadenu, and the LIC of the second patient normalized within 6 months of treatment.

\section{Conclusion}

Based on our observations, it is logical to screen patients with CLL and AIHA for iron overload, as this will help to avoid the various complications associated with iron deposition in different bodily organs. On the other hand, we observed that Jadenu is a safe and effective chelator with fewer side effects and better tolerability.

\section{Acknowledgments}

The authors would like to acknowledge Qatar National Library for supporting this publication and the Internal Medicine Residency Program at Hamad Medical Corporation for scientific support. 
Ali and Yassin: Iron Overload in Patients with CLL Complicated by AIHA

\section{Statement of Ethics}

Written informed consent was obtained from both patients to allow the publication of information including images. The case report was approved by the Hamad Medical Corporation Medical Research Center.

\section{Disclosure Statement}

The authors report no conflicts of interest regarding this work.

\section{Funding Sources}

The case report was funded by Qatar National Library.

\section{Author Contributions}

Both authors contributed equally.

\section{References}

1 Haskin G, Kogan M. Case report of unexpectedly long survival of patient with chronic lymphocytic leukemia: why integrative methods matter. Integr Med (Encinitas). 2018;17(1):51-6.

2 Zent CS, Kay NE. Autoimmune complications in chronic lymphocytic leukaemia (CLL). Best Pract Res Clin Haematol. 2010;23(1):47-59.

3 Yassin MA, Soliman A, De Sanctis V, Hmissi SM, Abdulla MA, Ekeibed Y, et al. The impact of iron overload in patients with acute leukemia and myelodysplastic syndrome on hepatic and endocrine functions. Acta Biomed. 2018;89(3-S):18-22.

4 Kanbour I, Chandra P, Soliman A, De Sanctis V, Nashwan A, Abusamaan S, et al. Severe liver iron concentrations (LIC) in 24 patients with $\beta$-thalassemia major: correlations with serum ferritin, liver enzymes and endocrine complications. Mediterr J Hematol Infect Dis. 2018;10(1):e2018062.

5 Yassin M, Soliman A, De Sanctis V, Nashwan A, Abusamaan S, Moustafa A, et al. Liver iron content (LIC) in adults with sickle cell disease (SCD): correlation with serum ferritin and liver enzymes concentrations in transfusion dependent (TD-SCD) and non-transfusion dependent (NT-SCD) patients. Mediterr J Hematol Infect Dis. 2017;9(1):e2017037.

6 Yassin MA, Soliman AT, De Sanctis V, Yassin KS, Abdulla MAJ. Final height and endocrine complications in patients with $\beta$-thalassemia intermedia: our experience in non-transfused versus infrequently transfused patients and correlations with liver iron content. Mediterr J Hematol Infect Dis. 2019;11(1):e2019026.

7 Lyle L, Hirose A. Iron overload in myelodysplastic syndromes: pathophysiology, consequences, diagnosis, and treatment. J Adv Pract Oncol. 2018;9(4):392-405.

8 Ambaglio I, Malcovati L, Papaemmanuil E, Laarakkers CM, Della Porta MG, Gallì A, et al. Inappropriately low hepcidin levels in patients with myelodysplastic syndrome carrying a somatic mutation of SF3B1. Haematologica. 2013;98(3):420-3.

9 Menchetti I, Lin Y, Cserti-Gazdewich C, Goldstein J, Law C, Lazarus A, et al. Complications of a severe autoimmune hemolytic anemia crisis: transfusional iron overload and gangrenous cholecystitis. Transfusion. 2018;58(12):2777-81.

10 Soliman AT, Al Yafei F, Al-Naimi L, Almarri N, Sabt A, Yassin M, et al. Longitudinal study on thyroid function in patients with thalassemia major: high incidence of central hypothyroidism by 18 years. Indian J Endocrinol Metab. 2013;17(6):1090-5.

11 De Sanctis V, Soliman A, Candini G, Campisi S, Anastasi S, Iassin M. High prevalence of central hypothyroidism in adult patients with $\beta$-thalassemia major. Georgian Med News. 2013;(222):88-94.

12 De Sanctis V, Soliman AT, Candini G, Yassin M, Raiola G, Galati MC, et al. Insulin-like growth factor-1 (IGF-1): demographic, clinical and laboratory data in 120 consecutive adult patients with thalassaemia major. Mediterr J Hematol Infect Dis. 2014;6(1):e2014074. 
13 De Sanctis V, Elsedfy H, Soliman AT, Elhakim IZ, Pepe A, Kattamis C, et al. Acquired hypogonadotropic hypogonadism $(\mathrm{AHH})$ in thalassaemia major patients: an underdiagnosed condition? Mediterr J Hematol Infect Dis. 2016;8(1):e2016001.

14 Soliman A, Yassin M, Al Yafei F, Al-Naimi L, Almarri N, Sabt A, et al. Longitudinal study on liver functions in patients with thalassemia major before and after deferasirox (DFX) therapy. Mediterr J Hematol Infect Dis. 2014;6(1):e2014025.

15 Yassin MA, Soliman AT, De Sanctis V, Hussein RM, Al-Okka R, Kassem N, et al. Jadenu ${ }^{\circledR}$ substituting Exjade ${ }^{\circledR}$ in iron overloaded $\beta$-thalassemia major (BTM) patients: a preliminary report of the effects on the tolerability, serum ferritin level, liver iron concentration and biochemical profiles. Mediterr J Hematol Infect Dis. 2018;10(1):e2018064. 